\title{
Perfil clínico e eletrorretinográfico da comoção retiniana
}

\author{
Clinical and electroretinographic profile of commotio retinae
}

\author{
Luciana da Cruz Noia ${ }^{1}$ \\ Adriana Berezovsky ${ }^{2}$ \\ Denise de Freitas ${ }^{3}$ \\ Paula Yuri Sacai ${ }^{4}$ \\ Solange Rios Salomãos
}

\begin{tabular}{|l|}
\hline RESUMO \\
\hline Objetivo: Traçar perfil clínico, anatômico e funcional da comoção re- \\
tiniana. Métodos: Pacientes com trauma ocular contuso e quadro fundos- \\
cópico de comoção retiniana unilateral há menos de 72 horas foram \\
submetidos ao exame de acuidade visual, biomicroscopia, oftalmoscopia \\
binocularindireta, retinografia, angiografia fluoresceínica, tomografia de \\
coerência óptica e eletrorretinografia de campo total. A eletrorretinografia \\
foi repetida após 15 e 30 dias. A angiografia após 30 dias. Resultados: \\
Foram incluídos 16 pacientes no estudo. No primeiro exame, havia \\
diferença estatisticamente significante entre o olho atingido e o olho \\
controle na amplitude de todas as respostas, sem alteração da relação \\
b/a, e na latência da resposta de cones isolados e do flicker a 30 Hz. No \\
segundo exame, manteve-se a diferença para os potenciais oscilatórios, \\
que desapareceu no último exame. Na angiofluoresceinografia, todos os \\
olhos acometidos mostravam áreas de hiperfluoresceência por transmis- \\
são alternadas com áreas de hipofluorescência por bloqueio. Este defeito \\
mantém-se após 30 dias. A tomografia de coerência óptica mostrou \\
diminuição da refletividade na camada dos fotorreceptores. Conclusões: \\
As alterações encontradas refletiram acometimento de fotorreceptores \\
e de células ganglionares, mas não da camada de células bipolares, além \\
de mobilização precoce de pigmento do epitélio pigmentado da retina. As \\
alterações eletrorretinográficas desapareceram após 30 dias do trauma. \\
\hline
\end{tabular}

Descritores: Eletrorretinografia; Traumatismos oculares; Retina; Angiofluoresceinografia; Tomografia de coerência óptica
Trabalho realizado no Laboratório de Eletrofisiologia Visual Clínica do Departamento de Oftalmologia da Universidade Federal de São Paulo - UNIFESP.

${ }^{1}$ Pós-graduanda, nível doutorado, em Oftalmologia pela Universidade Federal de São Paulo - UNIFESP - São Paulo (SP) - Brasil.

${ }^{2}$ Professora adjunta-doutora do Departamento de Oftalmologia da UNIFESP - São Paulo (SP) - Brasil.

Professora livre docente afiliada do Departamento de Oftalmologia da UNIFESP - São Paulo (SP) - Brasil.

${ }^{4}$ Pós-graduanda, nível mestrado, em Ciências Visuais pela UNIFESP - São Paulo (SP) - Brasil.

5 Professora adjunta-doutora do Departamento de Oftalmologia da UNIFESP - São Paulo (SP) - Brasil.

Endereço para correspondência: Rua Itapeva, 518 Cj. 1207/1208 - São Paulo (SP) CEP 01332-904 E-mail: lunoia@oftalmo.epm.br

Recebido para publicação em 28.11.2005

Última versão recebida em 03.05.2006

Aprovação em 14.05.2006

Apoio: FAPESP nº 97/11493-3 para Solange Rios Salomão; Bolsa de Doutorado pela CAPES, para Luciana da Cruz Noia, número de matrícula 7.215.108-9.

Nota Editorial: Depois de concluída a análise do artigo sob sigilo editorial e com a anuência do Dr. Maurício Bastos Pereira sobre a divulgação de seu nome como revisor, agradecemos sua participação neste processo.

\section{INTRODUÇÃO}

Lesões traumáticas oculares são a principal causa de perda visual unilateral em pacientes com idade entre 22 e 44 anos de idade $^{(1)}$. O último levantamento da "National Society to Prevent Blindness", feito em 1980, estima que ocorram anualmente 2 milhões e 400 mil traumas oculares ${ }^{(2)}$.

Contusão é um dos tipos mais freqüentes de trauma, alternando o primeiro lugar com corpo estranho superficial ${ }^{(3-7)}$.

O trauma ocular contuso pode causar lesão direta na parte anterior do olho: roturas radiais do esfíncter iriano, iridodiálise, recessão angular, ciclodiálise, rasgos no trabeculado, ruptura de zônula e diálise retiniana ${ }^{(8)}$. No segmento posterior, costumam ocorrer lesões indiretas, ou por contragolpe, como a comoção retiniana, a ruptura coriorretiniana posterior e o buraco macular traumático.

A comoção retiniana é a lesão vitreorretiniana mais comum nos traumas oculares contusos, aparecendo em $20,8 \%$ dos $\operatorname{casos}^{(9)}$. Foi descrita inicialmente por Berlin em 1873 e designava uma diminuição imediata da acuidade visual, com embaçamento retiniano oftalmoscopicamente visível, que se 
resolve espontaneamente sem deixar seqüela, com retorno da acuidade visual aos níveis pré-traumáticos ${ }^{(10)}$.

Atualmente o termo comoção retiniana é também utilizado para lesões localizadas perifericamente, mesmo que haja alteração permanente da acuidade visual ou do aspecto fundoscópico e o termo edema de Berlin designa um subgrupo que acomete o pólo posterior e não deixa seqüelas ${ }^{(11)}$.

Apresenta-se como uma opacificação branco-acinzentada, de morfologia geométrica, de margens freqüentemente maldefinidas. Os vasos retinianos são vistos claramente, e parecem inalterados. Pode estar associado a outras lesões retinianas como hemorragias sub-retinianas, intraretinianas ou préretinianas, rotura coroidal e descolamento seroso de retina ${ }^{(11)}$.

A acuidade visual costuma variar de 20/20 a 20/400, mas pode não se correlacionar ao grau de comprometimento visível da retina. É inclusive muito freqüente a baixa da acuidade visual preceder a lesão fundoscópica. $\mathrm{O}$ paciente pode estar assintomático, se a comoção retiniana estiver limitada à periferia da retina ${ }^{(12)}$.

Experimentos com porcos ${ }^{(13)}$, macacos ${ }^{(14)}$ e dados anatomo patológicos em seres humanos ${ }^{(15)}$, mostraram que a principal alteração encontrada é o rompimento das camadas externas dos fotorreceptores.

As alterações retinianas aparecem geralmente em topografia simetricamente oposta ao local do impacto ${ }^{(11)}$, sendo portanto o exemplo típico de lesão por contragolpe. O dano aos fotorreceptores é provavelmente causado por deformação do vítreo e forças hidráulicas ${ }^{(15)}$.

Há escassez de dados na literatura quanto ao uso de exames eletrofisiológicos para a avaliação da comoção retiniana. Os trabalhos que utilizaram eletrorretinografia em trauma ocular ou não descrevem a presença desta lesão específica ${ }^{(16-17)}$, ou são muito superficicais na análise dos dados obtidos ${ }^{(18-19)}$.

O objetivo deste estudo é de investigar as alterações clínicas, angiofluoresceinográficas, da tomografia de coerência óptica e eletrorretinográficas em olhos que sofreram trauma ocular contuso com comoção retiniana, nas primeiras 72 horas após o trauma. Em alguns casos foi possível o acompanhamento seriado da evolução dessas alterações.

\section{MÉTODOS}

Foram recrutados 16 pacientes que se apresentaram no Pronto Socorro de Oftalmologia do Hospital São Paulo - Universidade Federal de São Paulo no período de 8 de outubro de 2001 a 21 de outubro de 2004 e que preencheram os seguintes critérios:

- Trauma ocular contuso unilateral há menos de 72 horas;

- Olho contralateral com acuidade visual de 20/20, ou melhor.

Foram excluídos os pacientes que apresentassem opacidades de meios que impedisse a avaliação de detalhes da retina, patologias oculares prévias significantes relatadas ou encontradas e doenças sistêmicas.

Os pacientes que concordaram em colaborar com o estudo assinaram um termo de consentimento livre e esclarecido. O trabalho seguiu os princípios previstos na declaração de Helsinque e foi aprovado pelo Comitê de Ética em Pesquisa da Universidade Federal de São Paulo/ Hospital São Paulo.

\section{Exame oftalmológico}

A acuidade visual foi medida em tabela retro-iluminada, do tipo ETDRS ${ }^{(20)}$, Early Treatment Diabetic Retinopathy Study mostrada à distância de $4 \mathrm{~m}$. Os valores de acuidade foram considerados como logaritmo do mínimo ângulo de resolução (LogMAR).

Os pacientes foram submetidos à avaliação de defeito pupilar aferente, motilidade ocular extrínseca, palpação de rebordos orbitários a procura de sinais que pudessem indicar fratura de órbita, como irregularidade do rebordo ou crepitação, ao exame externo das pálpebras, biomicroscopia, tonometria de aplanação e oftalmoscopia binocular indireta.

Quando a lesão se encontrava posterior à região do equador, fazia-se uma retinografia do pólo posterior de ambos os olhos e fotografava-se toda a área esbranquiçada. A extensão da lesão foi calculada através da impressão destas retinografias. A área encontrada foi dividida pela área da papila.

\section{Angiografia fluoresceínica}

Os pacientes que não apresentaram contra-indicação e que concordaram com o procedimento foram submetidos à angiografia fluoresceínica também dentro de 72 horas, sempre após a eletrorretinografia. O exame foi repetido cerca de 30 dias após o trauma.

\section{Tomografia de coerência óptica (OCT)}

Os pacientes com lesão no pólo posterior foram submetidos à tomografia de coerência óptica. Foram realizados cortes ópticos lineares seriados de 2,83 a 5,92 mm de extensão, passando por uma porção de retina afetada e uma região de retina aparentemente não afetada. Os cortes ópticos foram interpolados e eventualmente alinhados, quando isso melhorasse a avaliação das camadas sem alterar artificialmente a arquitetura esperada da região.

\section{Eletrorretinografia (ERG)}

O exame foi realizado no Laboratório de Eletrofisiologia Visual Clínica do Departamento de Oftalmologia da Universidade Federal de São Paulo - UNIFESP, utilizando o sistema eletrodiagnóstico UTAS E-3000 (LKC Technologies, Inc., Gaithersburg, USA), com amplificação de 10.000 vezes e filtros eletrônicos variando de 0,3 a $500 \mathrm{~Hz}$. As amplitudes das respostas foram medidas em microvolts $(\mu \mathrm{V})$ e o tempo de culminação em milissegundos (ms).

Os pacientes tiveram suas pupilas dilatadas com uma gota de tropicamida a $1 \%$ e uma gota de fenilefrina a $10 \%$. Após adaptação dos dois olhos ao escuro por um tempo mínimo de 30 minutos, foi feita a preparação final, com a colocação do eletrodo de lente de contato em ambiente escuro, iluminado 
apenas por uma fraca lanterna vermelha, a fim de manter o estado de adaptação ao escuro. Os pacientes submetidos à oftalmoscopia binocular indireta no mesmo dia do ERG fizeram o segundo exame pelo menos 3 horas após o primeiro, e tiveram um tempo de adaptação ao escuro de pelo menos 1 hora.

Todos os pacientes utilizaram eletrodos de lentes de contato bipolares, com filamento de ouro (GoldLens electrode, Doran Instruments, MD, USA), inseridas após anestesia com uma gota de colírio de tetracaína, com metilcelulose a $2 \%$ na superfície da lente de contato, para proteger a córnea. Um eletrodo terra de cúpula de ouro em forma de clipe foi colocado no lóbulo da orelha, preenchido com gel eletrolítico.

Seguiu-se o protocolo padronizado pela International Society for Clinical Electrophysiology of Vision (ISCEV), para ERG clínico, constituído de 5 respostas: resposta escotópica de bastonetes, resposta escotópica máxima, potenciais oscilatórios, resposta de cones adaptada à luz e flicker a $30 \mathrm{~Hz}^{(21)}$, conforme descrito previamente ${ }^{(22)}$.

O exame foi realizado até 72 horas após o trauma e repetido cerca de 15 e 30 dias após o trauma.

Os seguintes parâmetros do ERG foram avaliados: a amplitude da onda "a" foi medida da linha de base até seu pico negativo. A amplitude da onda " $b$ " foi medida do pico negativo da onda "a" até o pico positivo da onda "b", em $\mu \mathrm{V}$. Os valores assim obtidos na máxima resposta foram usados para calcular a relação b/a. O tempo de culminação da onda "b" foi medido do início do estímulo até o pico da onda "b", em ms.

\section{Métodos estatísticos}

As análises estatísticas foram realizadas através do programa estatístico NCSS 2001 e BioEstat 1.0. Foi calculada a média, desvio padrão, mediana e percentis 2,5 e 97,5 dos parâmetros obtidos.

Os valores do olho afetado foram comparados aos do olho contralateral $^{(23)}$. Foi aplicado o teste com sinais de Wilcoxon para dados não paramétricos, já que os dados eletrorretinográficos representam uma população de distribuição geralmente não normal ${ }^{(24)}$. O nível de significância adotado para todos estes testes foi de $5 \%(\mathrm{p}<0,05)$.

As comparações entre o primeiro, segundo e terceiro exame foram feitas pelo teste de Friedman para amostras não paramétricas, adotando-se como nível de significância $\mathrm{p}<0,05$. Usou-se como variável para este teste a diferença interocular relativa, calculada pela fórmula:

$$
\mathrm{DIR}=\frac{(\mathrm{OA}-\mathrm{OC})}{\mathrm{OC}} \times 100
$$

Sendo, DIR: Diferença interocular relativa; OA: Valor do parâmetro no olho afetado; OC: Valor do parâmetro no olho controle.

A correlação entre a área aparentemente afetada e os parâmetros do ERG foi feita pela correlação de Spearman, considerando-se significativo $\mathrm{p}<0,05$.

\section{RESULTADOS}

\section{Perfil epidemiológico}

Foram recrutados 16 pacientes que foram submetidos a um primeiro ERG. Dentre esses pacientes, 4 eram do sexo feminino e 12 do sexo masculino. Em quatro pacientes foi acometido o olho direito e 11 o olho esquerdo. As idades variaram entre 15 e 60 anos, com mediana de 23 anos. Em 4 casos, o olho foi atingido por soco, 6 por bola (5 de futebol e um de handebol), um caso por rolha de champanhe e no último por impacto do olho contra o painel em acidente automobilístico.

Os exames foram realizados entre 4 horas e 3 dias após o trauma, sendo a mediana de 2 dias. Onze pacientes repetiram o exame por volta do $15^{\circ}$ dia e sete pacientes repetiram o exame após cerca de um mês.

\section{Exame oftalmológico}

A acuidade visual inicial variou entre -0,1 logMAR (20/16) e $<1,0 \log$ MAR (Contagem de dedos a $20 \mathrm{~cm}$ ). A evolução das acuidades visuais individuais é mostrada na tabela 1 .

As lesões oculares associadas foram: laceração corneana de espessura parcial em um caso, desepitelização de córnea (2 casos), laceração conjuntival (2 casos) e hemorragia subconjuntival (1 caso), hifema (3 casos - 1 microscópico, 2 grau I), irite traumática (5 casos). No exame externo, havia edema palpebral, de variável intensidade em todos os casos e um dos pacientes apresentava também fratura de arco zigomático.

À oftalmoscopia, 3 olhos apresentaram hemorragia vítrea que não dificultou a discriminação de detalhes da retina, 2 apresentaram hemorragia sub-retiniana e um apresentou rotura retiniana periférica.

Dez pacientes apresentaram lesões posteriores ao equador, que puderam ser retinografadas. Estas lesões variaram de 13 a 40 áreas de papila, com média de 24,1 e mediana de 20,5 áreas papilares. As lesões restantes encontravam-se muito anteriores para serem adequadamente retinografadas.

\section{Angiografia fluoresceínica}

Foi realizada angiografia fluoresceínica em 10 pacientes no primeiro exame, e repetida em 3 pacientes cerca de 30 dias após o trauma.

Em todos os pacientes foram encontradas áreas de hiperfluorescência por defeito em janela alternadas com áreas de hipofluorescência em bloqueio, em maior ou menor grau, mas geralmente discretas, a partir do primeiro exame. Este defeito manteve-se no $30^{\circ}$ dia nos pacientes que repetiram o exame (Figura 1).

Em dois pacientes foi encontrada impregnação tardia do epitélio pigmentado da retina (EPR), porém, a acuidade visual final nestes dois pacientes não foi muito afetada (respectivamente 20/20-2 e 20/20-1), o que pode ser explicado pelo fato de tais lesões não atingirem a mácula. Pelo pequeno número, não é possível fazer nenhuma correlação estatística entre impregna- 


\begin{tabular}{|c|c|c|c|c|c|c|c|}
\hline \multicolumn{8}{|c|}{ Tabela 1. Evolução da acuidade visual (Snellen e LogMar) } \\
\hline \multicolumn{2}{|c|}{ Paciente } & \multicolumn{2}{|c|}{ Primeiro exame } & \multicolumn{2}{|c|}{ Segundo exame } & \multicolumn{2}{|c|}{ Terceiro exame } \\
\hline № & Olho & Snellen & LogMar & Snellen & LogMar & Snellen & LogMar \\
\hline \multirow[t]{2}{*}{1} & Caso & $20 / 40-3$ & 0,36 & $20 / 25-2$ & 0,14 & $20 / 20-2$ & 0,04 \\
\hline & Controle & $20 / 20$ & 0,00 & $20 / 20-2$ & 0,04 & $20 / 20-2$ & 0,04 \\
\hline \multirow[t]{2}{*}{2} & Caso & $20 / 25-2$ & 0,14 & $20 / 20-1$ & 0,02 & $20 / 20-1$ & 0,02 \\
\hline & Controle & $20 / 20-1$ & 0,02 & $20 / 20-1$ & 0,02 & $20 / 20-1$ & 0,02 \\
\hline \multirow[t]{2}{*}{3} & Caso & $20 / 40-3$ & 0,36 & $20 / 20-3$ & 0,06 & $20 / 20-4$ & 0,08 \\
\hline & Controle & $20 / 20-1$ & 0,02 & $20 / 20-1$ & 0,02 & $20 / 20-1$ & 0,02 \\
\hline \multirow[t]{2}{*}{4} & Caso & $20 / 20-2$ & 0,04 & $20 / 20-2$ & 0,04 & $20 / 20$ & 0,00 \\
\hline & Controle & $20 / 20-3$ & 0,06 & $20 / 20-1$ & 0,02 & $20 / 20$ & 0,00 \\
\hline \multirow[t]{2}{*}{5} & Caso & $20 / 32-3$ & 0,26 & $20 / 20-1$ & 0,02 & $20 / 20-1$ & 0,02 \\
\hline & Controle & $20 / 20$ & 0,00 & $20 / 20$ & 0,00 & $20 / 20$ & 0,00 \\
\hline \multirow[t]{2}{*}{6} & Caso & $20 / 20-3$ & 0,06 & $20 / 16$ & $-0,10$ & $20 / 16$ & $-0,10$ \\
\hline & Controle & $20 / 25-2$ & 0,14 & $20 / 10-4$ & $-0,14$ & $20 / 16$ & $-0,10$ \\
\hline \multirow[t]{2}{*}{7} & Caso & $20 / 16$ & $-0,10$ & $20 / 16$ & $-0,10$ & $20 / 16$ & $-0,10$ \\
\hline & Controle & $20 / 16$ & $-0,10$ & $20 / 16$ & $-0,10$ & $20 / 16$ & $-0,10$ \\
\hline \multirow[t]{2}{*}{8} & Caso & $20 / 160-2$ & 0,94 & $20 / 100-1$ & 0,72 & & \\
\hline & Controle & $20 / 20-1$ & 0,02 & $20 / 20$ & 0,00 & & \\
\hline \multirow[t]{2}{*}{9} & Caso & $20 / 20-2$ & 0,04 & $20 / 20-1$ & 0,02 & & \\
\hline & Controle & $20 / 20$ & 0,00 & $20 / 20-1$ & 0,02 & & \\
\hline \multirow[t]{2}{*}{10} & Caso & $20 / 20$ & 0,00 & $20 / 20$ & 0,00 & & \\
\hline & Controle & $20 / 20$ & 0,00 & $20 / 20$ & 0,00 & & \\
\hline \multirow[t]{2}{*}{11} & Caso & $20 / 20$ & 0,00 & $20 / 20-1$ & 0,02 & & \\
\hline & Controle & $20 / 20$ & 0,00 & $20 / 20$ & 0,00 & & \\
\hline \multirow[t]{2}{*}{12} & Caso & $20 / 32-1$ & 0,22 & & & & \\
\hline & Controle & $20 / 20$ & 0,00 & & & & \\
\hline \multirow[t]{2}{*}{13} & Caso & $20 / 20$ & 0,00 & & & & \\
\hline & Controle & $20 / 20$ & 0,00 & & & & \\
\hline \multirow[t]{2}{*}{14} & Caso & $C D$ a $20 \mathrm{~cm}$ & $>1,00$ & & & & \\
\hline & Controle & $20 / 20-2$ & 0,04 & & & & \\
\hline \multirow[t]{2}{*}{15} & Caso & $20 / 20$ & 0,00 & & & & 0,00 \\
\hline & Controle & $20 / 20$ & 0,00 & & & & $-0,08$ \\
\hline \multirow[t]{2}{*}{16} & Caso & $20 / 20-2$ & 0,04 & & & & \\
\hline & Controle & $20 / 20$ & 0,00 & & & & \\
\hline
\end{tabular}

ção do EPR e a amplitude ou latência das ondas do ERG de campo total, mas estes pacientes tiveram respectivamente a segunda e a décima maior diferença interocular relativa na onda "a" e a primeira e a décima quarta maior diferença interocular na onda “b”, na máxima resposta no primeiro exame (Figura 2).

Dois pacientes tinham hemorragia sub-retiniana visível à oftalmoscopia no momento do primeiro exame. Nestes pacientes, a angiografia fluoresceínica além de confirmar o diagnóstico, mostrando áreas de bloqueio da fluorescência de coróide pelo sangue, sem bloqueio da fluorescência da vasculatura retiniana, também revelou um vazamento tardio próximo à hemorragia. Tais pacientes tiveram respectivamente a décima quarta e a décima sexta maior diferença interocular na onda "a" e a sétima e a décima segunda maior diferença interocular na onda "b", na máxima resposta (Figura 3).

\section{Tomografia de coerência óptica}

Apenas quatro pacientes tiveram lesões suficientemente próximas à mácula que puderam ser tomografadas. Dois deles apresentaram hemorragia sub-retiniana, que foi traduzida no OCT por uma separação da retina neurossensorial do EPR, com sombreamento posterior, que atenuava o EPR. Nestes pacientes, não foi possível detectar qualquer alteração da refletividade da camada de fotorreceptores.

Outros dois pacientes apresentaram apenas comoção retiniana. Nos cortes feitos na transição entre a retina de aspecto normal e a retina de aspecto esbranquiçado, percebe-se uma diminuição progressiva da refletividade em direção à retina afetada (Figura 4).

\section{Eletrorretinografia}

A análise dos dados mostrou que houve uma redução de amplitude estatisticamente significante entre o olho atingido e o olho controle nas cinco etapas do protocolo padrão do ERG. Quanto ao tempo de culminação, houve um aumento (atraso) estatisticamente significante no olho com trauma com relação ao olho contralateral somente na resposta fotópica de cones e no flicker a $30 \mathrm{~Hz}$. A relação b/a mostrou-se comparável entre 


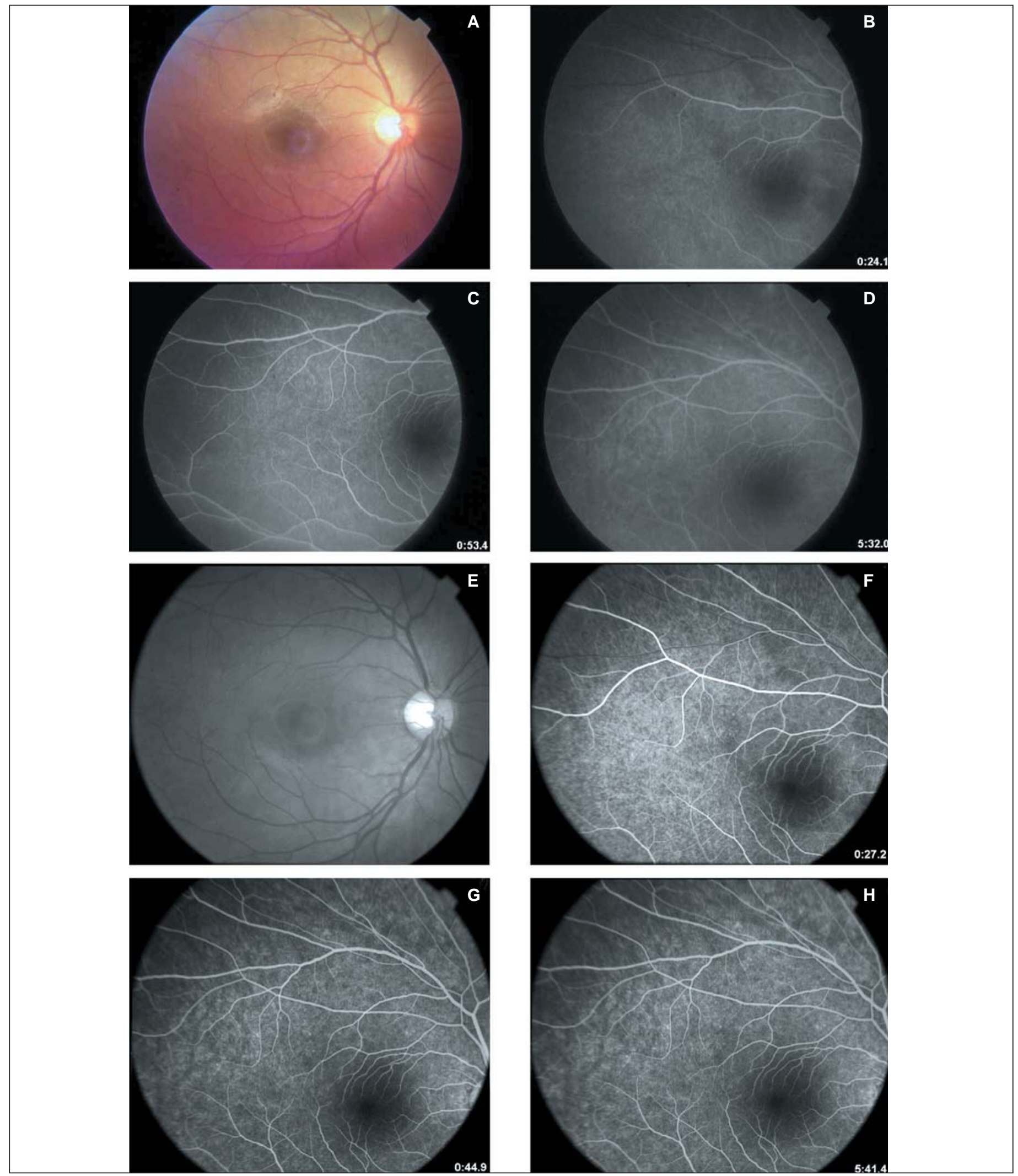

Figura 1 - Angiografia fluoresceínica do paciente ํㅜ 3, que sofreu contusão por bola de futebol há dois dias, mostrando discreto defeito em janela. Após 30 dias, repete-se o exame, manutenção do mesmo quadro angiográfico. A: retinografia colorida mostrando região esbranquiçada superior à mácula; $\mathrm{B}$ a $\mathrm{D}$ : angiofluoresceinografia do mesmo paciente, mostrando hiperfluorescência precoce que mantém limites em todo o exame, moteada com áreas de hipofluorescência por bloqueio; E: retinografia aneritra após 30 dias do trauma, mostrando fundo de olho normal; $\mathrm{F}$ a $\mathrm{H}$ : angiofluoresceinografia mostrando persistência do defeito em janela. 


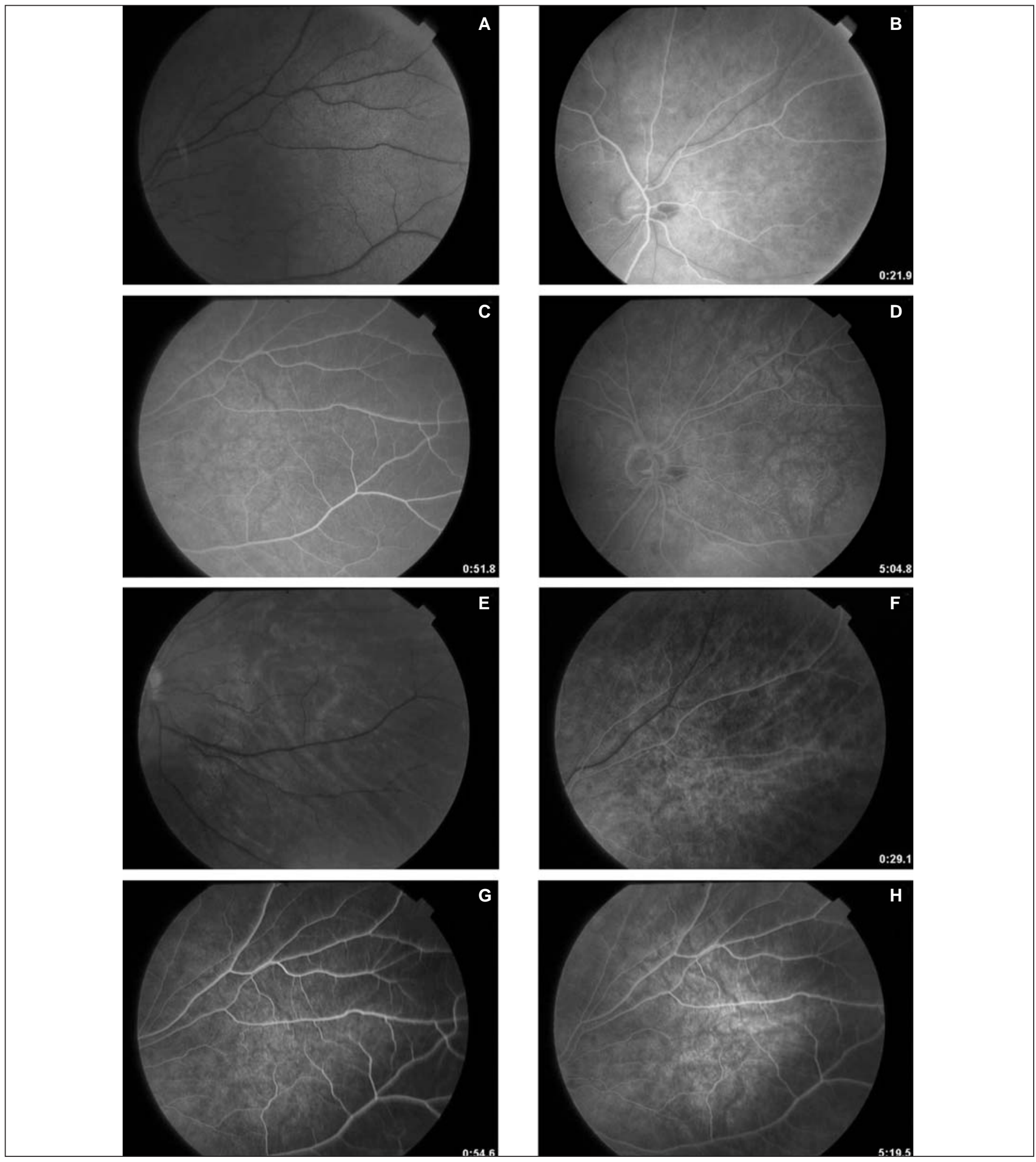

Figura 2 - Angiograria fluoresceínica do paciente $\mathrm{n}$-1, mostrando impregnação tardia do EPR e alterações pigmentares importantes. A: retinografia aneritra, obtida dois dias após o trauma ocular contuso, mostrando região esbranquiçada; B: fase venosa precoce, mostrando uma hiperfluorescência por defeito em janela; C: fase venosa tardia, mostrando a hiperfluorescência por defeito em janela e uma falha de enchimento da coróide, mostrando uma alteração na estrutura da microvascular da coriocapilar. D: fase de recirculação, mostrando persistência do padrão de fluorescência das fases anteriores; E: retinografia aneritra obtida 39 dias após o trauma, mostrando já mais claramente as alterações pigmentares coriorretinianas; F: fase venosa precoce, mostrando já a hiperfluorescência por defeito em janela; G: fase venosa tardia, mostrando áreas de hiperfluorescência e áreas de hipofluorescência por não perfusão (defeito da coriocapilar); H: fase de recirculação, mostrando manutenção do padrão de fluorescência das fases anteriores. 


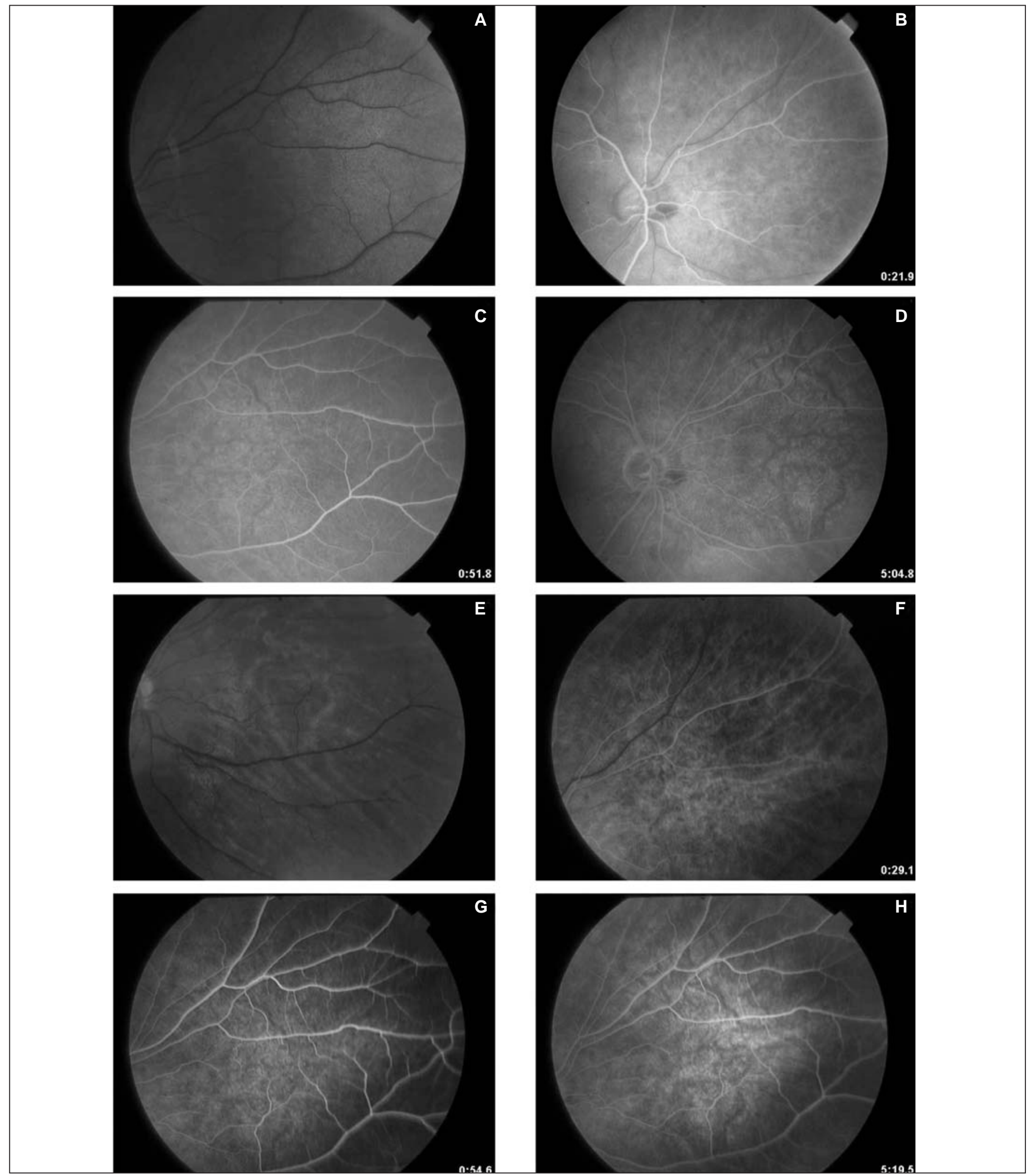

Figura 3 - Angiografia fluoresceínica do paciente oㅜ 8, dois dias após o trauma contuso, mostrando hemorragia sub-retiniana e vazamento tardio perilesional de contraste. A: retinografia colorida, mostrando hemorragia sub-retiniana em arcada temporal superior, próxima à mácula, rodeada de retina esbranquiçada (comoção retiniana); B: fase arterial, 18,2 segundos após a injeção de contraste, mostrando hiperflorescência por bloqueio nas áreas cobertas por hemorragia sub-retiniana, e uma discreta hiperfluorescência por defeito em janela na área correspondente à comoção retiniana; C: fase venosa tardia, 54,7 segundos após a injecão de contraste, mostrando início de ponto de vazamento de contraste na borda temporal da hemorragia; D: fase da recirculação: aumento da área de hipefluorescência por vazamento. 


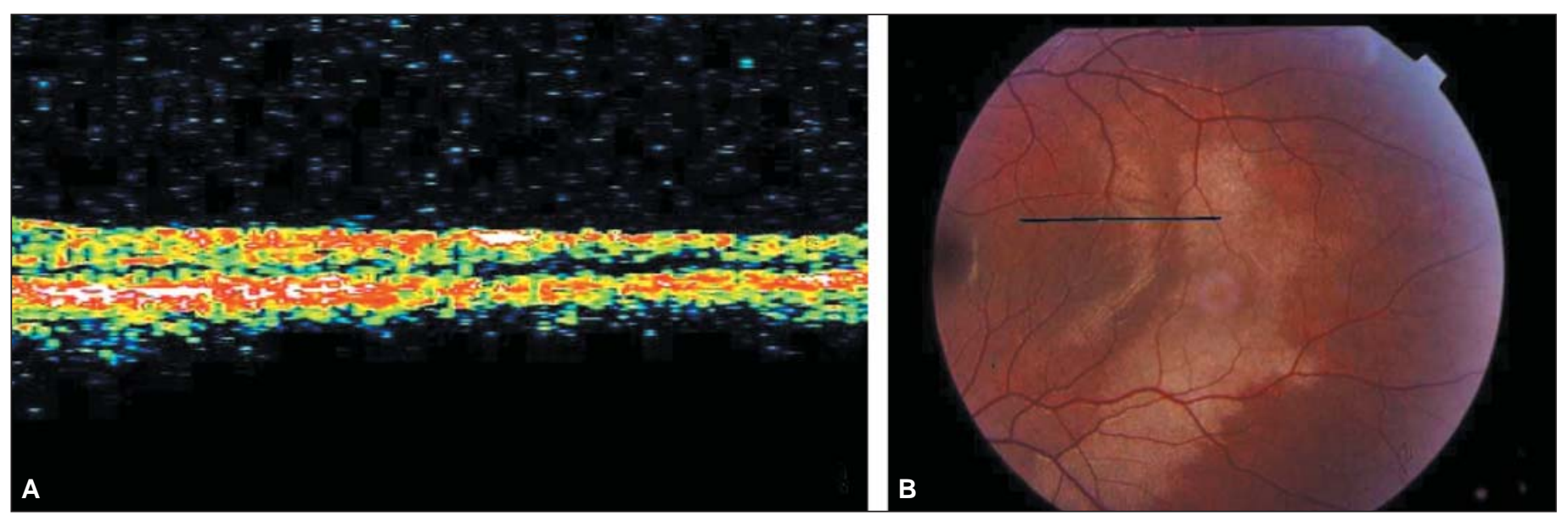

Figura 4 - Tomografia de coerência óptica de paciente com comoção retiniana. A: corte tomográfico alinhado e interpolado, mostrando vazio óptico na região de lesão visível; B: transposição da região do corte óptico para uma retinografia colorida, para uma melhor visibilização do corte em relação à lesão.

o olho com trauma e o olho contralateral, não havendo diferenças estatísticas (Tabela 2).

A redução foi mais evidente na amplitude da resposta escotópica de bastonetes ( $47 \%$ de redução da média em relação ao olho contralateral), que nas respostas puras de cones ( $13 \%$ de redução na resposta isolada de cones e $11 \%$ no flicker a $30 \mathrm{~Hz}$ ). As máximas respostas mantiveram uma redução da média intermediária entre as respostas de cones e bastonetes (30\% e $38 \%$ de redução das ondas "a" e "b", respectivamente). Os potenciais oscilatórios apresentaram uma diferença entre as médias de cerca de $30 \%$.

No segundo exame, manteve-se a diferença apenas dos potenciais oscilatórios. No último exame, não foram detectadas diferenças estatisticamente significantes para nenhum dos parâmetros (Tabela 2). A figura 5 mostra um exemplo da evolução das respostas do ERG de um dos pacientes do estudo.

A diferença interocular relativa de amplitude mostrou-se significantemente diferente entre o primeiro e o segundo exame, mas não entre o segundo e o terceiro exame na resposta escotópica de bastonetes $(\mathrm{p}=0,021)$ e na onda " $\mathrm{b}$ " da máxima resposta $(\mathrm{p}=0,018)$. Nos demais parâmetros que inicialmente apresentavam-se alterados, não houve diferença significativa desta diferença entre vários exames.

O teste de correlação de Spearman para amostras não paramétricas não mostrou correlação entre a área de lesão visível e a diminuição relativa do ERG (Tabela 3), em nenhuma das respostas.

\section{DISCUSSÃO}

Foram recrutados pacientes com trauma ocular unilateral, sem patologias oculares prévias relatadas ou encontradas ao exame ocular, para que o olho contralateral pudesse servir de controle e amenizer possíveis diferenças de amplitudes encontradas em pacientes de sexo e idade diferentes ${ }^{(23,25-27)}$.

Os pacientes deveriam realizar o ERG nas primeiras 72 horas após o trauma ocular contuso, pois existem evidências de que o maior acometimento das células da retina externa acontece nos três primeiros dias. Sipperley et al., descreveram uma diminuição do número de núcleos picnóticos na camada externa da retina no quarto dia após o trauma em macacos ${ }^{(14)}$.

\begin{tabular}{|c|c|c|c|c|c|c|c|c|c|c|c|}
\hline \multirow[t]{2}{*}{ Paciente } & \multirow[t]{2}{*}{ Olho } & \multicolumn{2}{|c|}{ Resposta escotópica } & \multicolumn{2}{|c|}{ Máxima resposta } & \multirow{2}{*}{$\begin{array}{c}\text { Relação } \\
\text { b/a }\end{array}$} & \multirow{2}{*}{$\begin{array}{l}\text { Potenciais } \\
\text { oscilatórios }\end{array}$} & \multicolumn{2}{|c|}{ Resposta fotópica } & \multicolumn{2}{|c|}{ Flicker } \\
\hline & & Amplitude & Latência & Onda a & Onda b & & & Amplitude & Latência & Amplitude & Latência \\
\hline $1^{\circ}$ exame & Afetado & 188,8 & 85,0 & 136,4 & 308,9 & 2,277 & 142,2 & 98,7 & 28,8 & 63,3 & 28,1 \\
\hline média & Controle & 277,8 & 84,3 & 178,1 & 425,2 & 2,450 & 185,2 & 111,4 & 28,2 & 70,4 & 27,4 \\
\hline $\mathrm{p}$ & & 0,0004 & 0,8753 & 0,0038 & 0,0005 & 0,4691 & 0,0032 & 0,0214 & 0,0329 & 0,0386 & 0,02 \\
\hline $2^{\circ}$ exame & Afetado & 244,0 & 88,2 & 153,2 & 391,1 & 2,666 & 147,6 & 93,3 & 29,4 & 55,8 & 28,3 \\
\hline média & Controle & 275,9 & 90,3 & 167,1 & 416,4 & 2,553 & 172,8 & 104,4 & 28,5 & 62,9 & 27,7 \\
\hline$p$ & & 0,0505 & 0,6891 & 0,286 & 0,2132 & 0,5049 & 0,0208 & 0,1307 & 0,1731 & 0,0619 & 0,1688 \\
\hline $3^{\circ}$ exame & Afetado & 230,4 & 85,8 & 142,3 & 363,6 & 2,549 & 133,8 & 102,4 & 29,3 & 62,5 & 27,8 \\
\hline média & Controle & 267,3 & 85,6 & 161,6 & 395,5 & 2,453 & 163,0 & 104,9 & 28,7 & 65,0 & 27,5 \\
\hline$p$ & & 0,063 & 0,9326 & 0,1282 & 0,1305 & 0,499 & 0,063 & 0,7353 & 0,3454 & 0,6121 & 0,398 \\
\hline
\end{tabular}


Hart mostrou que na maioria dos casos, a opacificação da retina persiste por 3 a 4 dias $^{(28)}$ o que confirma a observação inicial de Berlin, de que a lesão não costumava ser mais visível após 3 dias do trauma ${ }^{(10)}$.

A maioria dos pacientes foi do sexo masculino, numa proporção de 3 homens para cada mulher. Esta é semelhante à proporção clássica encontrada em trauma ocular ${ }^{(29-30)}$.

Em nosso estudo, 12 dos 16 pacientes (75\%) tinham menos de 30 anos. Dados do sistema de registro de trauma ocular americano também mostram que $58 \%$ dos pacientes tinham menos de $30 \operatorname{anos}^{(29)}$.

As acuidades visuais encontraram-se pouco diminuídas. Os casos de maior baixa de acuidade visual foram os associados à hemorragia sub-retiniana na região macular (casos 8 e 14). A literatura mostra que esta é uma causa importante de baixa de acuidade visual persistente após comoção retiniana ${ }^{(11,31)}$.

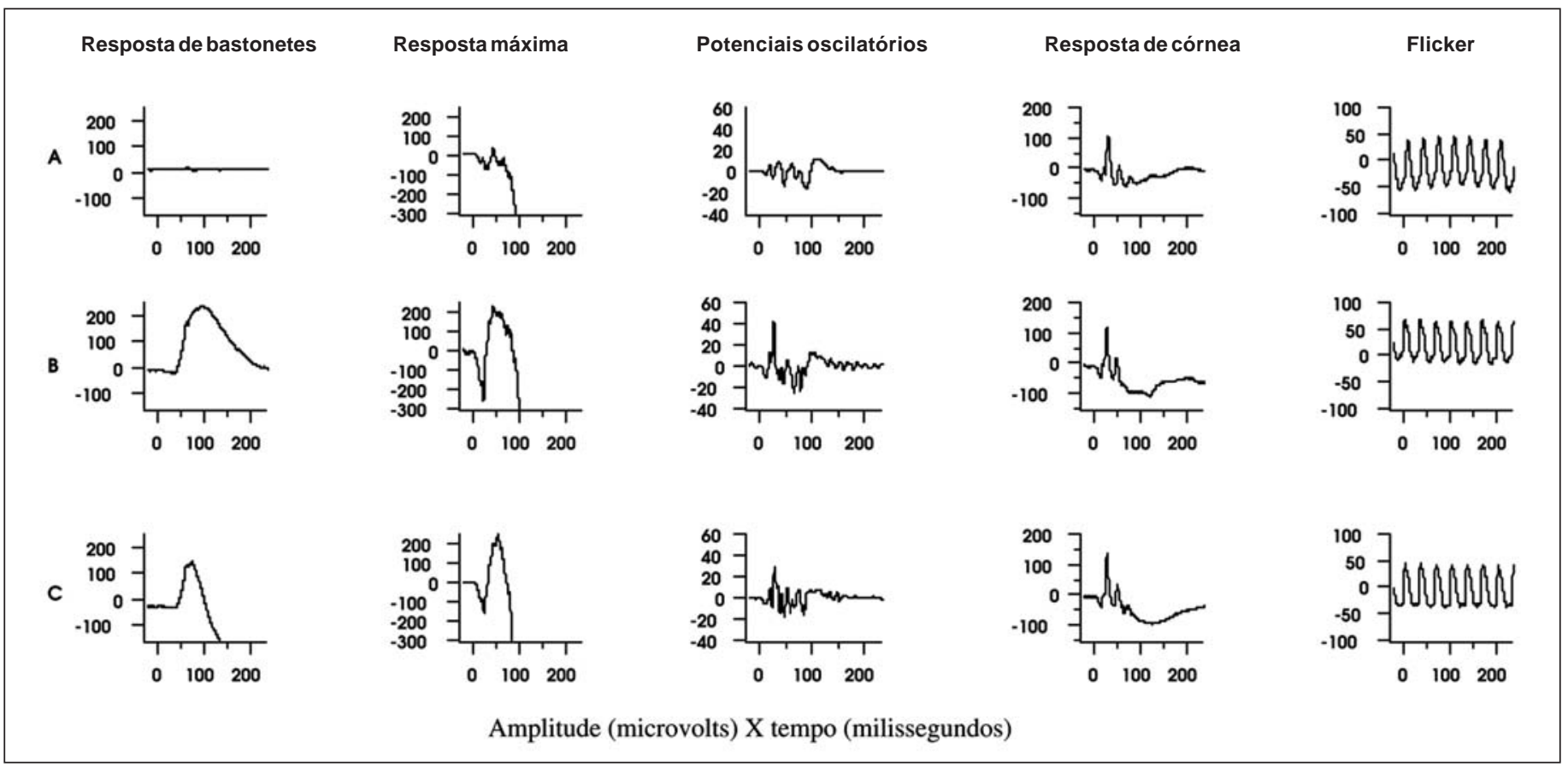

Figura 5 - Exemplo representativo da evolução das cinco respostas do eletrorretinograma de campo total do paciente no 1, de 18 anos, incluído no estudo. A: exame realizado dois dias após o trauma ocular contuso no olho direito; B: exame realizado 24 dias após o trauma ocular contuso no olho direito; C: exame realizado 39 dias após o trauma ocular contuso no olho direito.

\begin{tabular}{|c|c|c|c|c|c|c|c|c|c|c|c|}
\hline Paciente & DP & REBA & REBT & MRA & MRB & BA & PO & RCA & RCT & FLA & FLT \\
\hline 1 & 36,38 & 100 & 100 & 61,93 & 71,16 & 24,28 & 43,48 & 3,66 & $-12,50$ & $-6,16$ & $-16,09$ \\
\hline 2 & 23,18 & 22,70 & $-2,22$ & 22,62 & 14,15 & $-10,97$ & 8,13 & $-1,17$ & 0 & 4,99 & 1,08 \\
\hline 3 & 16,66 & 47,88 & $-2,98$ & 64,59 & 64,00 & $-1,69$ & 68,90 & 20,38 & $-4,92$ & 21,01 & $-8,42$ \\
\hline 4 & 17,82 & 40,05 & 0 & 26,92 & 23,60 & $-4,52$ & 3,06 & $-12,23$ & 4,68 & $-13,38$ & $-0,37$ \\
\hline 5 & 29,52 & 8,98 & $-1,16$ & 20,91 & 7,17 & $-17,39$ & 21,61 & 0,70 & 0 & $-12,95$ & 2,20 \\
\hline 9 & 15,30 & 56,03 & $-3,07$ & $-17,33$ & 39,66 & 48,55 & 42,06 & 32,96 & $-5,66$ & 20,22 & 0,37 \\
\hline 10 & 17,35 & 18,80 & 9,23 & $-7,33$ & $-1,18$ & 5,76 & $-26,82$ & $-10,10$ & $-1,85$ & $-21,98$ & 1,81 \\
\hline 12 & 13,11 & 41,18 & $-3,72$ & 19,15 & 17,56 & $-1,95$ & 10,13 & 19,57 & $-3,33$ & 20,46 & $-3,69$ \\
\hline 14 & 31,33 & 29,63 & $-3,52$ & $-16,83$ & 14,28 & 26,63 & 14,08 & 23,34 & 0 & 25,91 & $-3,48$ \\
\hline 15 & 40,43 & 46,36 & 8,78 & 37,24 & 31,43 & $-9,21$ & 41,68 & 21,98 & 0 & 4,81 & $-1,39$ \\
\hline $\begin{array}{l}\text { Média } \\
\text { Mediana }\end{array}$ & $\begin{array}{l}20,11 \\
20,50\end{array}$ & & & & & & & & & & \\
\hline \multirow{2}{*}{\multicolumn{2}{|c|}{$\begin{array}{l}\text { Coeficiente } \\
\text { de Spearman } \\
p\end{array}$}} & $-0,0545$ & 0,5515 & 0,3455 & 0,0424 & $-0,2242$ & 0,1515 & 0,0061 & 0,3455 & $-0,2$ & $-0,0545$ \\
\hline & & 0,881 & 0,0983 & 0,3282 & 0,9074 & 0,5334 & 0,6761 & 0,9867 & 0,3282 & 0,5796 & 0,881 \\
\hline \multicolumn{12}{|c|}{ 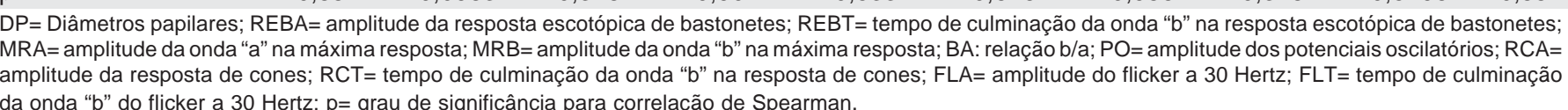 } \\
\hline
\end{tabular}


As lesões oculares mais freqüentemente encontradas foram as lesões de câmara anterior (irite traumática e hifema), que são as lesões mais encontradas em qualquer trauma ocular contuso ${ }^{(32)}$. As alterações à oftalmoscopia indireta (hemorragia vítrea, hemorragia sub-retiniana e rotura retiniana) também são descritas como freqüentes ${ }^{(32-33)}$.

Desde o primeiro exame, mesmo que realizado apenas algumas horas após o trauma, houve áreas de hiperfluorescência por defeito de transmissão alternadas com áreas de hipofluorescência por bloqueio. Este achado pode ser interpretado como resultado da heterogeneidade da distribuição da melanina no EPR traumatizado.

A migração de pigmento do EPR é descrita em estudos anatomo-patológicos experimentais. Blight descreveu o encontro ocasional de material derivado do EPR em macrófagos ${ }^{(13)}$. Sipperley notou áreas de perturbação do EPR com defeito em janela associado em macacos que sofreram comoção retinia$\mathrm{na}^{(14)}$. O mesmo autor explica este achado como consequiência da migração precoce de células epiteliais através das camadas da retina, encontrada em cortes histológicos da retina dos mesmos animais ${ }^{(14)}$.

No grupo estudado, o OCT mostrou vazio óptico na região acometida, que aumentava conforme o corte progredia da região de coloração normal para a região esbranquiçada. Esses achados são idênticos aos de estudo anterior que descreve OCT em um paciente com comoção retiniana ${ }^{(34)}$. Este vazio pode ser a tradução óptica da classicamente descrita disrupção da camada externa dos fotorreceptores ${ }^{(13-15,35)}$.

No primeiro exame eletrorretinográfico encontramos diferenças estatisticamente significantes nas amplitudes de bastonetes e cones, bem como no flicker. O tempo de culminação estava aumentado nas respostas de cones (resposta fotópica de cones e flicker), mas não na resposta escotópica de bastonetes. Tal fato talvez seja explicado por ser esta resposta mais lenta, portanto menos sujeita a alterações. Também sua grande variabilidade $^{(22)}$ torna difícil detectar tais alterações.

Estes achados não podem ser atribuídos a opacidades de meios, já que todos os pacientes os apresentavam transparentes, no máximo com hemorragia vítrea discreta em três pacientes (Tabela 1). Jayle, Tassy demonstraram, através da interposição de vidros contendo sangue humano total, que até $1 \mathrm{~mm}$ de sangue causa pouca alteração na amplitude do ERG com flashes de luz brancos ${ }^{(16)}$.

$\mathrm{Na}$ análise da máxima resposta, houve diminuição tanto da onda "a" quanto da onda "b". A onda "a" reflete a atividade de fotorreceptores $^{(36)}$ e a onda "b" das células de Müller, conseqüente à passagem do impulso pelas células bipolares ${ }^{(37)}$. Quando há uma diminuição da onda "a", há uma proporcional diminuição da onda "b" pela diminuição do impulso que chega às células bipolares. A relação b/a, entretanto, fica preservada.

Como no nosso estudo não houve alteração da relação b/a em relação ao olho contralateral, conclui-se que a diminuição da onda "a" é o evento primário refletindo uma perda funcional da camada de fotorreceptores ${ }^{(36)}$, que encontra respaldo nos resultados anátomo-patológicos em olhos de porcos ${ }^{(13)}$, de $\operatorname{macacos}^{(14)}$ e humanos ${ }^{(15)}$. Nestes trabalhos, as principais alterações encontradas estavam concentradas na interface entre o EPR e a camada externa da retina.

A relação b/a preservada também torna improvável um componente isquêmico importante, como já suspeitava Hart et al. Em um de seus trabalhos, notou que a extinção da onda "b" do ERG dava-se logo após o trauma, enquanto que na isquemia, tal extinção acontece minutos após a oclusão da veia central ${ }^{(18)}$.

As alterações dos potenciais oscilatórios podem estar relacionadas a ação das forças hidrodinâmicas na interface vítreoretiniana, provocando uma lesão por cisalhamento dos axônios da camada de fibras nervosas, à semelhança da lesão axonal difusa cerebral. A ação dessas forças de desaceleração no trauma ocular contuso pode ser demonstrada pelo descolamento de vítreo posterior, encontrado em maior proporção em olhos traumatizados ${ }^{(38)}$.

Embora nenhuma alteração morfológica tenha sido encontrada no modelo experimental com macacos de Sipperley ${ }^{(14)}$, outros trabalhos mostram indícios de lesão a este nível.

Mansour et al., em estudo de microscopia eletrônica em olho humano eviscerado após menos de 24 horas de trauma, com comoção retiniana, encontrou edema mitocondrial discreto em retina interna nas áreas acometidas ${ }^{(15)}$.

Hart et al., também descreveu axônios mais eletrodensos que o normal, com aspecto de compressão, inclusões císticas no axoplasma e edema das mitocôndrias na camada de fibras nervosas, em modelo experimental em olhos de porco ${ }^{(18)}$. Estes achados morfológicos poderiam também justificar a diminuição dos potenciais oscilatórios encontrada no presente estudo.

O mecanismo pelo qual a comoção retiniana acontece é denominado classicamente lesão por contragolpe ${ }^{(39)}$. Por este mecanismo, forças hidráulicas transmitidas posteriormente, associadas a uma deformação do vítreo ${ }^{(15,40-41)}$ causariam um movimento relativo entre tecidos de densidades diferentes ${ }^{(42)}$.

Assim, os tecidos mais propensos a sofrerem o efeito do impacto seriam as interfaces entre o vítreo e a retina e entre o EPR e os fotorreceptores. As alterações dos potenciais oscilatórios e da amplitude da onda "a" refletem este efeito. As outras camadas da retina não sofreriam a ação dessa deformação, pois estariam ligadas pelas células de Müller ${ }^{(15)}$. A preservação da relação b/a mostra que embora a onda b esteja diminuída, isto acontece por causa da diminuição da onda "a", e não por um efeito direto na função das células de Müller, confirmando o pouco efeito do trauma ocular contuso sobre as mesmas.

No entanto, as lesões não foram tão extensas a ponto de justificar uma redução tão grande da média da amplitude da resposta de bastonetes atingidos $(188,8 \mu \mathrm{V})$ em relação ao olho controle $(277,8 \mu \mathrm{V})$, o que indica que não apenas a área esbranquiçada, mas uma boa parte da retina aparentemente inalterada apresentavam algum grau de diminuição das respostas.

O quadro clínico de comoção retiniana (esbranquiçamento das camadas externas da retina) parece, portanto, traduzir apenas um grau maior de uma alteração que acomete a retina submetida a essas ondas de choque como um todo, como sugeriu Hart 
et al. ${ }^{(18)}$. A lesão esbranquiçada representaria apenas um acometimento acima de um certo limiar, mas uma parte muito maior da retina apresenta-se funcionalmente comprometida.

Progressivamente, as diferenças encontradas no ERG foram diminuindo, não sendo mais estatisticamente significantes aos 30 dias após o trauma, fazendo-nos crer que o ritmo da evolução é lento, mas a recuperação tende a ser completa.

$\mathrm{Na}$ análise estatística, entretanto, houve alteração da diferença entre o primeiro e o segundo exame e apenas nos parâmetros amplitude da resposta escotópica de bastonetes e amplitude da onda "b" na máxima resposta. Tal fato se deu porque este estudo não foi desenhado para determinar este dado, uma vez que por ser a primeira descrição de ERG de campo total em olhos humanos com comoção retiniana, não existiam dados prévios para se calcular o tamanho da amostra necessária para se obter tal resposta. Com os dados obtidos por este estudo, este cálculo poderá ser feito, possibilitando um futuro aprofundamento desta análise.

\section{CONCLUSÃO}

Os dados do presente estudo mostram que as lesões causadas pela comoção retiniana são reversíveis, por afetarem estruturas capazes de se regenerar, como os segmentos externos dos fotorreceptores, ou pela própria natureza reversível da alteração anatômica, como é o caso do edema mitocondrial, axoplasmático e intracelular. Provavelmente algumas células sofreriam lesão acima deste limiar, podendo chegar até a morte celular. Elas não voltariam a contribuir para a corrente elétrica registrada pelo eletrorretinograma, mas sua proporção na retina dos pacientes analisados poderia ter sido muito pequena, de forma que não interfeririam na recuperação da resposta total do ERG.

\section{AGRADECIMENTO}

Pela ajuda em todas as fases desse trabalho a Josenilson Martins Pereira, pós-graduando do Departamento de Oftalmologia da UNIFESP.

\section{ABSTRACT}

Purpose: To investigate clinical, anatomic and electroretinographic changes in eyes that suffered blunt ocular trauma with commotio retinae. Methods: Patients who presented commotio retinae after unilateral blunt ocular trauma less then 72 hours before were submitted to visual acuity testing, biomicroscopy, binocular indirect ophthalmoscopy, fluorescein angiography, optical coherence tomography and full-field electroretinography. Full-field ERG was repeated after 15 and 30 days. Results: Sixteen patients were included in the study. On the first examination there was a statistically significant difference between affected and fellow eye in all response amplitudes, without b/a ratio alteration, and a delay in singleflash cone response and $30-\mathrm{Hz}$ flicker implicit time. On the second examination, the difference between the eyes remained for oscillatory potentials, but disappeared on the last examination. In fluorescein angiography, all patients presented mottled hyperfluorescence and hypofluorescent areas, due to alterations in the pigment barrier. On optical coherence tomography, we found optically empty spaces at the site of the lesion. Conclusions: Found changes suggested photoreceptor and ganglion cells, but not Müller cell functional alterations, as well as pigment mobilization. These changes disappeared 30 days after the trauma.

Keywords: Electroretinography; Eye injuries; Retina; Fluorescein angiography; Optical coherence tomography

\section{REFERÊNCIAS}

1. Congdon NG, Friedman DS, Lietman T. Important causes of visual impairment in the world today. JAMA. 2003;290(15):2057-60.

2. National Society to Prevent Blindness: Vision Problems in the USA: a statistical analysis. New York: National Society to Prevent Blindness; 1980.

3. Desai P, MacEwen C, Baines P, Minassian D. Epidemiology and implications of ocular trauma admitted to hospital in Scotland. J Epidemiol Community Health 1996;50(4):436-41.

4. Voon LW, See J, Wong TY. The epidemiology of ocular trauma in Singapore: perspective from the emergency service of a large tertiary hospital. Eye. 2001;15(Pt 1):75-81.

5. Tzelikis PF de M, Diniz CM, Alvim H da S, Gonçalves RM, Araújo Júnior AR de, Trindade FC. Perfil do paciente com trauma ocular atendido no Hospital São Geraldo da Universidade Federal de Minas Gerais. Rev Bras Oftalmol. 2002;61(12):885-91.

6. Bernucci EA, Lopreto RCC, Rodrigues M de LV. Traumatismos oculares em uma unidade de emergência. Rev Bras Oftalmol. 1993;52(6):407-11.

7. Andrade AS de, Bisneto OS, Moreira H, Moreira CA. Traumas oculopalpebrais no serviço de pronto-atendimento oftalmológico da Hospital Universitário Evangélico de Curitiba. Arq Bras Oftalmol. 1999;62(5):585-9.

8. Campbell D. Traumatic glaucoma. In: Shingleton BJ, Hersh PS, Kenyon KR, editors. Eye trauma. St. Louis: Mosby Year Book; 1991.

9. Tozatti MSV, Moraes NS, Farah ME, Uno F, Barbosa LB, Vianna RC. Freqüência de lesões vitreorretinianas pós-trauma contuso. Arq Bras Oftalmol. 1993;56(4): 168 .

10. Berlin R. Zur sogenannten Commotio retinae. Klin. Monatsbl. Augenheilkd. $1873 ; 11: 42-78$

11. Bressler SB, Bressler NM. Traumatic maculopathies. In: Shingleton BJ, Hersh PS, Kenyon KR, editors. Eye trauma. St. Louis: Mosby Year Book; 1991.

12. Benson WE, Jeffers JB. Blunt trauma. In: Tasman W, Jaeger EA, editors. Duane's Clinical Ophthalomology. Philadelphia: JB Lippincott; 1998.

13. Blight R, Hart JC. Structural changes in the outer retinal layers following blunt mechanical non-perforating trauma to the globe: an experimental study. Br J Ophthalmol. 1977;61(9):573-87.

14. Sipperley JO, Quigley HA, Gass DM. Traumatic retinopathy in primates. The explanation of commotio retinae. Arch Ophthalmol. 1978;96(12):2267-73.

15. Mansour AM, Green WR, Hogge C. Histopathology of commotio retinae. Retina. 1992;12(1):24-8.

16. Jayle GE, Tassy AF. Prognostic value of the electroretinogram in severe recent ocular trauma. Br J Ophthalmol. 1970;54(1):51-8.

17. Zelentsov SN, Iakovlev AA, Stepanov AV. [Clinical-functional state of the retina and optic nerve in contusions of the eyeball] Vestn Oftalmol. 1995;111 (4):12-4. Russian.

18. Hart JC, Blight R, Cooper R, Papakostopoulos D. Electrophysiological and pathological investigation of concussional injury. An Experimental study. Trans Ophthalmol Soc U K. 1975;95(2):326-34.

19. Purvin V, Maturi R, Vaphiades MS. Sprint car visual loss. Surv Ophthalmol 2004;49(1):90-5.

20. Ferris FL 3rd, Kassoff A, Bresnick GH, Bailey I. New visual acuity charts for clinical research. Am J Ophthalmol. 1982;94(1):91-6. 
21. Marmor MF, Holder GE, Seeliger MW, Yamamoto S. International Society for Clinical Electrophysiology of Vision. Standard for clinical electroretinography (2004 update). Doc Ophthalmol. 2004;108(2):107-14.

22. Pereira JM, Mendieta L, Sacai PY, Salomão SR, Berezovsky A. Estudo normativo do eletrorretinogramam de campo total em adultos jovens. Arq Bras Oftalmol. 2003;66(2):137-44.

23. Van Lith G. Subnormal and absent ERGs: what do we mean by these terms? Doc Ophthalmol. 1982;31:13-7.

24. Marmor MF, Zrenner E. Standard for clinical electroretinography (1999 update). International Society for Clinical Electrophysiology of Vision. Doc Ophthalmol. 1998-99;97(2):143-56.

25. Zeidler I. The clinical electroretinogram. IX. The normal electroretinogram. Value of the b-potential in different age groups and its differenes in men and women. Acta Ophthalmol (Copenh). 1959;37:294-301.

26. Peterson $\mathrm{H}$. The normal B-potential in the single-flash clinical electroretinogram. A computer technique study of the influence of sex and age. Acta Ophthalmol (Copenh). 1968;Suppl 99:7-77.

27. Birch DG, Anderson JL. Standardized full-field electroretinography. Normal values and their variation with age. Arch Ophthalmol. 1992;110(11):1571-6.

28. Hart JC, Frank HJ. Retinal opacification after blunt non-perforating concussional injuries to the globe. A clinical and retinal fluorescein angiographic study. Trans Ophthalmol Soc U K. 1975;95(1):94-100.

29. May DR, Kuhn FP, Morris RE, Witherspoon CD, Danis RP, Matthews GP, Mann L. The epidemiology of serious eye injuries from the United States Eye Injury Registry. Graefes Arch Clin Exp Ophthalmol. 2000;238(2):153-7.

30. Wong TY, Klein BE, Klein R. The prevalence and 5 year incidence of ocular trauma. The Beaver Dam Eye Study. Ophthalmology. 2000;107(12):2196-202.

31. Dugel PU, Ober RR. Posterior segment manifestations of closed-globe contusion injury. In: Ryan SJ, editor. Retina. St. Louis: Mosby; 2001. p.2386-99.
32. Eagling EM. Ocular damage after blunt trauma to the eye. Its relationship to the nature of the injury. Br J Ophthalmol. 1974;58(2):126-40.

33. Williams DF, Mieler WF, Williams GA. Posterior segment manifestations of ocular trauma. Retina. 1990;10 Suppl 1:S35-44.

34. Meyer CH, Rodrigues EB, Mennel S. Acute commotio retinae determined by cross-sectional optical coherence tomography. Eur J Ophthalmol. 2003;13 (9-10):816-8.

35. Hui YN, Wu YQ, Xiao QS, Kirchhof B, Heimann K. Repair of outer bloodretinal barrier after severe ocular blunt trauma in rabbits. Graefes Arch Clin Exp Ophthalmol. 1993;231(6):365-9.

36. Brown KT, Wiesel TN. Localisation of origins of electroretinogram components by intraretinal recording in the intact cat eye. J Physiol (Lond) 1961; 158:257-80.

37. Newman EA, Odette LL. Model of electroretinogram b-wave generation: a test of the K+ hypothesis. J Neurophysiol 1984;51(1):164-82.

38. Wedrich A, Velikay M, Binder S, Radax U, Stolba U, Datlinger P. Ocular findings in asymptomatic amateur boxers. Retina. 1993;13(2):114-9.

39. Diniz AV, Freitas JAH, Carvalho JOT, Barsante C. Manifestações do trauma ocular contuso. In: Abujamra S, Ávila M, Barsante C, Farah ME, Gonçalves JOR, Lavinsky J, et al., editors. Retina e vítreo - Clínica e cirurgia. São Paulo: Roca; 2000. p.769-77.

40. Weidenthal DT, Schepens CL. Peripheral fundus changes associated with ocular contusion. Am J Ophthalmol. 1966;62(3):465-77.

41. Delori F, Pomerantzeff O, Cox MS. Deformation of the globe under high speed impact: Its relation to contusion injuries. Invest Ophthalmol. 1969;8 (3):290-301

42. Courville CB. Coup-contrecoup mechanism of cranio-cerebral injuries. Arch Surg. 1942;45:19-43. 